\title{
Real-time monitoring of small animal cortical hemodynamics by photoacoustic tomography
}

Changhui Li, Andres Aquirre, John Gamelin, Anastasios Maurudis, Quing Zhu, et al.

Changhui Li, Andres Aquirre, John Gamelin, Anastasios Maurudis, Quing Zhu, Lihong V. Wang, "Real-time monitoring of small animal cortical hemodynamics by photoacoustic tomography," Proc. SPIE 7564, Photons Plus Ultrasound: Imaging and Sensing 2010, 756427 (23 February 2010); doi: $10.1117 / 12.842159$

SPIE. Event: SPIE BiOS, 2010, San Francisco, California, United States 


\title{
Real-time monitoring of small animal cortical hemodynamics by photoacoustic tomography
}

\author{
Changhui Li ${ }^{a}$, Andres Aguirre ${ }^{b}$, John Gamelin ${ }^{b}$, Anastasios Maurudis ${ }^{b}$, Quing Zhu ${ }^{b}$, and \\ Lihong V. Wang ${ }^{a}$ \\ ${ }^{a}$ Optical Imaging Laboratory, Department of Biomedical Engineering Washington University \\ in St.Louis, St.Louis, Missouri 63130; \\ ${ }^{b}$ Department of Electrical \& Computer Engineering, University of Connecticut, Storrs, \\ Connecticut 06269
}

\begin{abstract}
For the first time, the hemodynamics within the entire cerebral cortex of a mouse were studied by using photoacoustic tomography (PAT) non-invasively and in real time. The PAT system, based on a 512-element full-ring array with cylindrical focusing, received the PA signal primarily from a slice of about $2 \mathrm{~mm}$ thickness. This system can provide not only high resolution brain vasculature images but also hemodynamic functional images. We recorded the wash-in process of a photoacoustic contrast agent in a mouse brain in real time. Our results demonstrated that PAT is a powerful imaging modality to study real-time small animal neurofunctional activities that cause changes in hemodynamics.
\end{abstract}

Keywords: Photoacoustic imaging, hemodynamics, small animal imaging, neurofunctional imaging

\section{INTRODUCTION}

Photoacoustic (PA) tomography (PAT) is a hybrid of optics and ultrasound. ${ }^{1-3}$ Based on the photoacoustic effect, which transfers the absorbed optical radiation energy into mechanical pressure, PAT reconstructed the image of optical absorbers by detecting the PA pressure waves. Unlike X-ray imaging, PAT uses non-ionizing radiation source, and can image tissue non-invasively and in vivo. Compared with other high resolution pure optical imaging methods, such as confocal microscopy, two-photon microscopy, and optical coherence tomography (OCT), PAT breaks through their common imaging barrier ( $1 \mathrm{~mm}$ under skin) to image targets within the diffusive regime at a high ultrasonic resolution. Over the past two decades, this imaging modality has gained significant progresses in biomedicine. ${ }^{4,5}$

Photoacoustic (PA) tomography (PAT) has successfully imaged small animal cortex. ${ }^{6-9}$ However, the imaging speeds of most of the previous PAT brain systems are slow, making it challenging to study fast hemodynamic changes in the entire cortex. For instance, a PAT system based on the scanning of a single element generally needs more than ten minutes to finish one complete data acquisition. ${ }^{6}$ In this work, we presented that for the first time the hemodynamics within the entire cortex of a mouse were monitored by PAT in real time. The fast PAT system is based on a 512-element full-ring array, which provides an PA imaging frame rate up to 1 $\mathrm{Hz}$, i.e., one image per second. ${ }^{10}$ The dramatically increased imaging speed enables real-time PA monitoring of physiological processes, such as the monitoring of the uptake of PA contrast as described later.

In this paper, we first introduced the real-time PAT system and the contrast agent that was used in the animal experiment. Then, we monitored the uptake process of the agent in the cortical vasculature of a mouse non-invasively and in real time, discussed the experimental results. After that, we provided a summary.

Further author information: (Send correspondence to Changhui Li) Changhui Li: E-mail: cli@biomed.wustl.edu, Telephone: (314)935-9586

Photons Plus Ultrasound: Imaging and Sensing 2010, edited by Alexander A. Oraevsky, Lihong V. Wang, Proc. of SPIE Vol. 7564,756427 - (C) 2010 SPIE · CCC code: 1605-7422/10/\$18 - doi: 10.1117/12.842159 


\section{REAL-TIME PAT SYSTEM}

The PAT imaging system uses a 512-element ultrasonic array. Each element is cylindrically focused, leading to a imaging slice of about $2 \mathrm{~mm}$ thickness. The diameter of the array is $5 \mathrm{~cm}$, and the field of view is about 1.2 $\mathrm{cm}$ in diameter. The center frequency of the detector is about $5 \mathrm{MHz}$, and the bandwidth is greater than $80 \%$. The spatial resolution within the imaging plane is less than $200 \mu \mathrm{m}$. Although PA signals from all 512 channels have been preamplified parallely, the second-stage amplifiers have only 64 channels. A 8:1 multiplier was used, and total 8 acquisitions are required for an complete full ring acquisition. Moreover, the limited speed in data transfer requires the temporal interval between two successive acquisitions a bit greater than $0.125 \mathrm{~s}$. Thus, a optimal laser repetition rate is between 7 to $8 \mathrm{~Hz}$, enabling the frame rate of the system close to $1 \mathrm{~Hz}$. However, for a higher repetition rate laser, relative low data transfer speed results in missed laser shorts between data acquisitions. For instance, the repetition rate of the laser source used in this paper is $10 \mathrm{~Hz}$, only every other laser short actually contributed to the data acquisition, leading to a reduced frame rate to $0.625 \mathrm{~Hz}$, i.e., $1.6 \mathrm{~s}$ per image. The imaging speed can be increased by either adding more channels or improving the data transfer speed. More technical details about this PAT system is described in. ${ }^{10}$

We also studied the stability of current system, which was done by monitoring the temporal variations in PA images of a static target. A black human hair was chosen, which was fixed in the imaging plane. Another thin wire ring was used as a reference object, whose PA signal amplitude was used for the compensation of variations in the laser pulse power and system sensitivity. Our experimental results shows the PAT imaging system has a $5 \%$ instability. The stability can be further improved by using more sensitive photodiode to measure the laser pulse power and reduce the electrical noise in the data acquisition system.

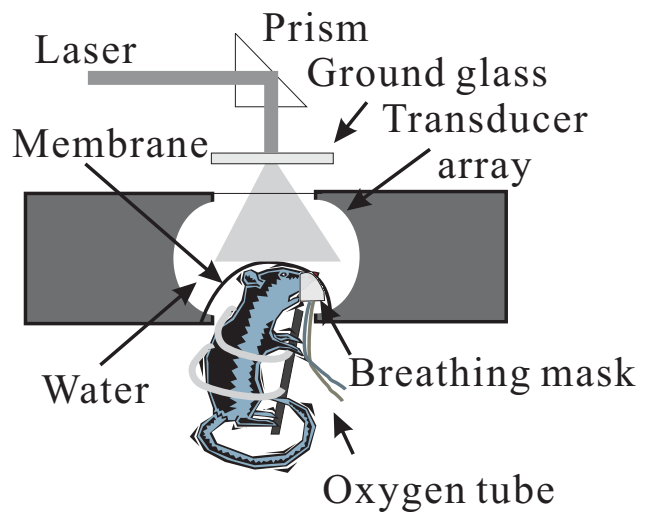

Figure 1. Side view of the array system and the handling of the animal.

\section{CONTRAST AGENT}

To demonstrate the real-time imaging capability, we monitored the uptake process of Evans blue dye in small animal cortical vasculature. Evans blue was traditionally used for measuring the total blood volume since it tightly binds with blood albumin. In addition, this dye has an optical absorption peak around $620 \mathrm{~nm},{ }^{11}$ where the blood absorption is relatively low. Thus, a significant increase in PA signal is expected after injecting the dye by using the dye's high absorption spectrum. Moreover, the fast uptake of Evans blue makes it a good model for real-time imaging. In our experiments, the dye was administrated via the tail vein injection, and the PAT imaged the cortical vasculature at $620 \mathrm{~nm}$ wavelength.

\section{IN VIVO EXPERIMENTS}

The cerebral cortex of a Swiss Webster mouse (Harlan Sprague Dawley Incorporated, Indianapolis, Indiana, $24 \mathrm{~g}$ ) was imaged in vivo by PAT. Figure 1 shows the experimental setup. The hair on the mouse head was gently depilated, and the mouse was fixed on a homemade animal holder. After applying a thin layer of ultrasound gelatine, the mouse head protruded up to the imaging plane, against a thin transparent plastic membrane. Before the tail vein injection, we imaged the cortical vasculature at $532 \mathrm{~nm}$ and $620 \mathrm{~nm}$, as seen in Fig. 2(a, b). The 
imaged cortical vasculature is consistent with the anatomical photograph. As expected, the image obtained by using $620 \mathrm{~nm}$ has a much lower contrast than that by using $532 \mathrm{~nm}$.

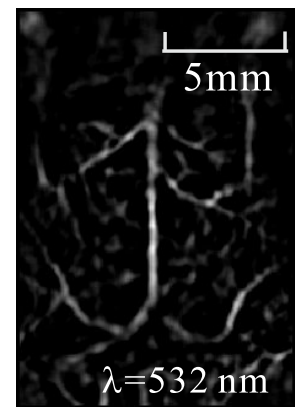

(a)

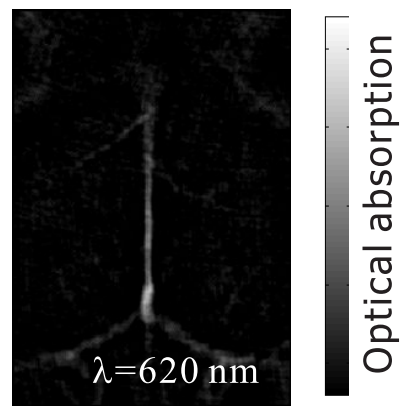

(b)

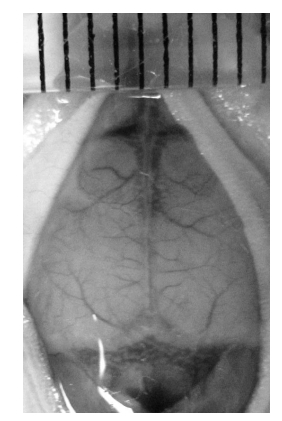

(c)

Figure 2. (a) Reconstructed PAT images at $532 \mathrm{~nm}$ wavelength. (a) Reconstructed PAT images at $620 \mathrm{~nm}$ wavelength. (c) The anatomical photography of the cortex. The photograph was taken with the scalps removed after the mouse was euthanized.

We then monitored the uptake of Evans blue at $620 \mathrm{~nm}$ wavelength. About $0.2 \mathrm{~mL} 3 \%$ Evans blue was administrated by tail vein injection, which start around $30 \mathrm{~s}$ and took about $40 \mathrm{~s}$. PAT continually recorded the PA signal at one image per $1.6 \mathrm{~s}$. Then, images were reconstructed off line. Figure 3(a) shows the reconstructed images at different times. During the experiment, the animal was anesthetized by adding $1 \%$ isoflurane in the oxygen supply. All experimental animal procedures conformed to the National Institutes of Health (NIH) guidelines and were in compliance with the Washington University Institutional Animal Care and Use Committee (IACUC).

As we expected, without contrast dye, the contrast in PAT image is low. The Evans blue significantly increased the PA signal and thus recovered more vascular structures. In addition, the fast imaging speed enabled the real-time monitoring the hemodynamics over the entire cortical region. By normalization image values by that at $\mathrm{t}=0 \mathrm{~s}$, Fig. 3 (c) plots the relative PA absorption within a selected region that is marked by dashed white square. Our results shows the optical absorption increased about $180 \%$ after the injection.

\section{CONCLUSIONS}

In summary, real-time in vivo PAT has been demonstrated in imaging the entire cortical vasculature of small mice. The temporal resolution of in our experiment is about $1.6 \mathrm{~s}$ per image, which is hundreds of times faster than most of previous PA brain imaging systems. The imaging speed can be further improved by adding more channels or reducing the data transfer time. Because cortical hemodynamic parameters, including blood volume and oxygen saturation, are closely associated with brain functions, our results demonstrated that PAT has a great potential to aid the study of neurofunctional activities non invasively. 

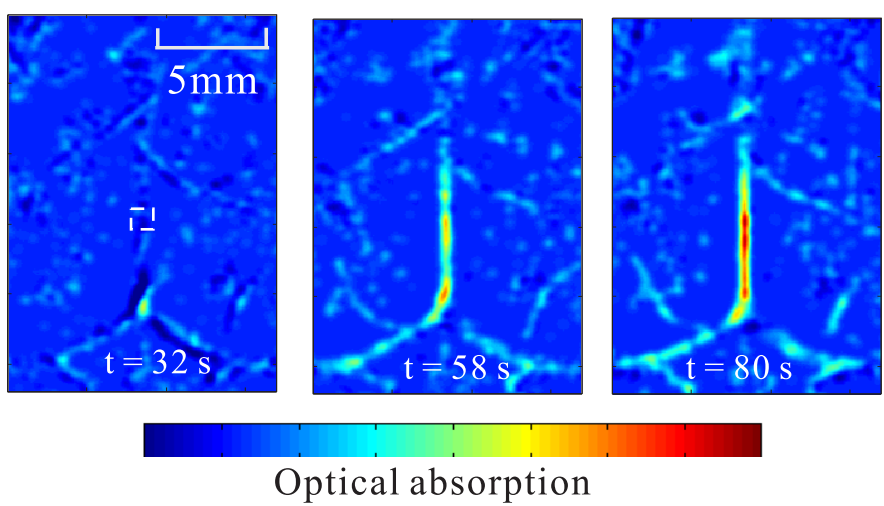

(a)

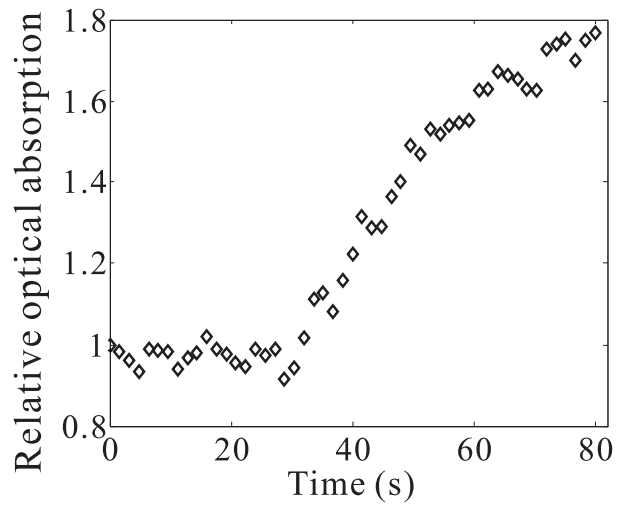

(b)

Figure 3. (Color online) Real-time PAT results at $620 \mathrm{~nm}$ wavelength. (a) Reconstructed PA images at t $=32 \mathrm{~s}, 58 \mathrm{~s}$, and $80 \mathrm{~s}$, respectively. (b) The relative PA absorption change vs. time.

\section{Acknowledgments}

We thank the Yu Wang and Junjie Yao for helpful discussions. This work has been supported in part by National Institutes of Health grants R01 EB000712, R01 EB008085, and U54 CA136398 (Network for Translational Research). L.W. has a financial interest in Microphotoacoustics, Inc. and Endra, Inc., which, however, did not support this work.

\section{REFERENCES}

[1] Oraevsky, A. and Karabutov, A., "Optoacoustic tomography," in [Biomedical Photonics Handbook], VoDinh, T., ed., CRC, Boca Raton, FL (2003).

[2] Wang, L. V. and Wu, H.-i., [Biomedical Optics : Principles and Imaging], Wiley, Hoboken, N.J. (2007).

[3] Wang, L. V., ed., [Photoacoustic Imaging and Spectroscopy], CRC Press, Boca Raton, 1 ed. (2009).

[4] Xu, M. and Wang, L. V., "Photoacoustic imaging in biomedicine," Review of Scientific Instruments 77, 41101-41122 (2006).

[5] Li, C. and Wang, Lihong, V., "Photoacoustic tomography and sensing in biomedicine," Physics in Medicine and Biology 54, R59 (2009).

[6] Wang, X. D., Pang, Y. J., Ku, G., Xie, X. Y., Stoica, G., and Wang, L. H. V., "Noninvasive laser-induced photoacoustic tomography for structural and functional in vivo imaging of the brain," Nature Biotechnology 21(7), 803-806 (2003).

[7] Stein, E. W., Maslov, K., and Wang, L. H. V., "Noninvasive, in vivo imaging of the mouse brain using photoacoustic microscopy," Journal of Applied Physics 105(10), 5 (2009). 
[8] Yang, S., Xing, D., Zhou, Q., Xiang, L., and Lao, Y., "Functional imaging of cerebrovascular activities in small animals using high-resolution photoacoustic tomography," Medical Physics 34(8), 3294-3301 (2007).

[9] Laufer, J., Zhang, E., Raivich, G., and Beard, P., "Three-dimensional noninvasive imaging of the vasculature in the mouse brain using a high resolution photoacoustic scanner," Applied Optics 48, D299-D306 (2009).

[10] Gamelin, J., Maurudis, A., Aguirre, A., Huang, F., Guo, P., Wang, L. V., and Zhu, Q., "A real-time photoacoustic tomography system for small animals," Opt. Express 17(13), 10489-10498 (2009).

[11] Patterson, C. E., Rhoades, R. A., and Garcia, J. G. N., "Evans blue-dye as a marker of albumin clearance in cultured endothelial monolayer and isolated lung," Journal of Applied Physiology 72, 865-873 (1992). 